\title{
Formation and structure of the three Neptune-mass planets system around HD 69830
}

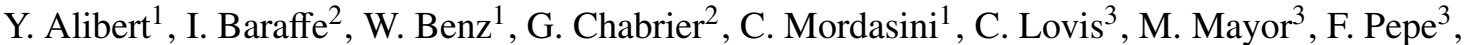 \\ F. Bouchy ${ }^{4}$, D. Queloz ${ }^{3}$, and S. Udry ${ }^{3}$ \\ 1 Physikalisches Insitut, University of Bern, Sidlerstrasse 5, 3012 Bern, Switzerland \\ e-mail: yann. alibert@space.unibe.ch \\ 2 CRAL, École Normale Supérieure, 46 allée d'Italie, 69007 Lyon, France \\ 3 Observatoire de Genève, 51 Ch. des Maillettes, 1290 Sauverny, Switzerland \\ 4 Institut d'Astrophysique de Paris, 98bis Bd Arago, 75014 Paris, France
}

Received 26 May 2006 / Accepted 6 July 2006

ABSTRACT

\begin{abstract}
Since the discovery of the first giant planet outside the solar system in 1995 (Mayor \& Queloz 1995, Nature, 378, 355), more than 180 extrasolar planets have been discovered. With improving detection capabilities, a new class of planets with masses 5-20 times larger than the Earth, at close distance from their parent star is rapidly emerging. Recently, the first system of three Neptune-mass planets has been discovered around the solar type star HD 69830 (Lovis et al. 2006, Nature, 441, 305). Here, we present and discuss a possible formation scenario for this planetary system based on a consistent coupling between the extended core accretion model and evolutionary models (Alibert et al. 2005a, A\&A, 434, 343; Baraffe et al. 2004, A\&A, 419, L13; Baraffe et al. 2006, A\&A, 450, 1221). We show that the innermost planet formed from an embryo having started inside the iceline is composed essentially of a rocky core surrounded by a tiny gaseous envelope. The two outermost planets started their formation beyond the iceline and, as a consequence, accrete a substantial amount of water ice during their formation. We calculate the present day thermodynamical conditions inside these two latter planets and show that they are made of a rocky core surrounded by a shell of fluid water and a gaseous envelope.
\end{abstract}

Key words. stars: planetary systems - stars: planetary systems: formation

\section{Introduction}

The three Neptune-mass planetary system orbiting HD69830, a 4-10 Gyr old nearby star with a mass estimated at $0.86 \pm$ $0.03 M_{\odot}$, has been discovered through high precision measurements obtained with the HARPS spectrograph installed at $\mathrm{La}$ Silla, Chile (Lovis et al. 2006). The three planets, planets b,c and d, are located at $0.0785,0.186$ and $0.63 \mathrm{AU}$ from the central star, and their minimum masses are equal to $10.2,11.8,18.1 M_{\oplus}$ respectively. This system, with three sub-Neptune mass planets within $1 \mathrm{AU}$, represents a considerable challenge for planet formation models, namely the disk instability (DI) model and the core-accretion (CA) model.

\section{Formation by disk instability}

In the DI model, gravitational instabilities directly lead to the formation of clumps that eventually evolve to form giant planets. In the case of the present planetary system, this formation mechanism can be ruled out for two reasons. First, the inner regions of the disk are too hot for gravitational instabilities to take place. Second, gravitational instabilities at larger distances produce clumps with masses much larger than those considered here (e.g. Boss 2001). Hence, even if subsequent migration brings these clumps within $1 \mathrm{AU}$, they would be much more massive than the planets considered here. Indeed, at least for the two outermost planets, we show that mass loss from evaporation induced by the host star's high energy radiation is negligible.
It has been suggested that low mass planets could form in the framework of the DI model, assuming the presence of a FUV/EUV source close to the formation site (e.g. a close-in O star) that would evaporate the gas envelope of initially larger mass planets (Boss 2006). If such an external source is present, photoevaporation indeed occurs at distances from the central star larger than $r_{\mathrm{e}} \propto G M_{\text {star }} / c_{\mathrm{s}}^{2}$, where $M_{\text {star }}$ is the mass of the central star, and $c_{\mathrm{s}}$ is the sound speed of gas heated up by the FUV/EUV flux (Boss 2006). Estimations of $r_{\mathrm{e}}$ depend on the energy of the incoming flux, and range from $5 \mathrm{AU}$ for EUV to $50 \mathrm{AU}$ for FUV (Johnstone et al. 1998). In the case of the Solar System, these values are consistent with a possible evaporation of Uranus and Neptune, and the preservation of Jupiter's and Saturn's gaseous envelopes. Given that the mass of HD 69830 is close to solar, the photoevaporation radius $r_{\mathrm{e}}$ can be expected to be similar as well. If this is correct, the photo-evaporation of planet $\mathrm{d}$ located well inside $r_{\mathrm{e}}$ appears quite impossible. However, other calculations point out that $r_{\mathrm{e}}$ could be as low as 1 AU for EUV flux and $10 \mathrm{AU}$ for FUV flux (Adams et al. 2004). If these calculations are correct, the evaporation of planet $d$ by an external source would be marginally possible. However, in this case the survival of Jupiter's atmosphere becomes a problem unless one argues that evaporation took place due to EUV flux in the case of the HD 69830 system, and due to FUV flux in the case of the Solar System. Furthermore, we note that reconciling the DI/photoevaporation model and the present system requires evaporation to start after the outermost planet has reached a location close to its present location (inward migration of planets 
formed by DI at large distances requires a substantial amount of gas). Finally, at a given EUV flux, a planet evaporates increasingly faster as its mass diminishes (Baraffe et al. 2004, 2006). Hence, to account for planet d requires a very fine tuning between the start and stop of the evaporating flux. While not impossible, such special circumstances appear quite unlikely. Finally, we note that DI has been also strongly excluded as a possible formation mechanism of the HD 149026 system (Sato et al. 2005). The discovery of a Jupiter mass planet orbiting at yet larger distances from HD 69830 would definitely rule out the $\mathrm{DI} /$ photoevaporation as a possible formation mechanism, at least for this particular system.

\section{Formation by nucleated instability}

In the CA model, a solid core is first formed by the accretion of solid planetesimals. When its mass is large enough, it can accrete gas in a runaway process, rapidly building up a giant planet (Alibert et al. 2005a; Hubickyj et al. 2005). In the framework of this model, the in situ formation of cores large enough to trigger the runaway gas accretion, and consequently the in situ formation of close-in giant planets, is prevented by the sheer lack of solid material that close to the star. On the one hand, low mass disks simply lack the necessary amount of solids, while on the other hand more massive disks with similar lifetimes are too hot for solids to condense at these short distances. Thus, to reach their present mass, the planets orbiting HD 69830 must have swept planetesimals over distant regions of the disk. Therefore, the discovery of this system of hot-Neptune planets implies (if there were any doubts left) that significant planetary migration had to occur.

In order to compute the formation and the evolution of this system, we use the extended CA model which takes into account the migration of the proto-planets as well as the evolution of the disk. We also consider the evolution of the new born planets to the present day by taking into account the effects of irradiation and evaporation due to stellar radiation. Our entire approach has been extensively described elsewhere (Alibert et al. 2005a; Baraffe et al. 2004, 2006), where the reader is referred for more details. Using these models, we performed a large number (few tens of thousand) of simulations to find all initial conditions leading to a planetary system comparable to this one. Assuming a central star of $0.86 M_{\odot}$ and a dust-to-gas ratio of $1 / 70$, accounding for the slightly sub-solar metallicity of the star (Lovis et al. 2006), we start our calculations with a protoplanetary disk and seed the three planets by means of three embryos of $0.6 M_{\oplus}$ each. We explore the different disk characteristics (mass and lifetime) and initial locations of the three embryos leading to the observed characteristics of the three planets. Finally, note that we do not take into account gravitational interactions between the three forming planets that could alter the migration rates. However, we have checked that the planets do not cross their mutual main mean motion resonances during the formation (in particular the 1:2,1:3 and 2:3 resonances).

Since planets migrate significantly during their growth, they eventually encounter the wake created by the preceeding planet. To account for this, we considered the formation of the planets from inside out, each embryo being started at a different distance and time and followed while migrating through a disk already modified by the preceding planets. As the planet encounters a region depleted of planetesimals by the passage of a previous body, the accretion rate of solids is vanishing, leading to the suppression of the main heating source and the planet can accrete gas (Alibert et al. 2005b) at a rate essentially given by

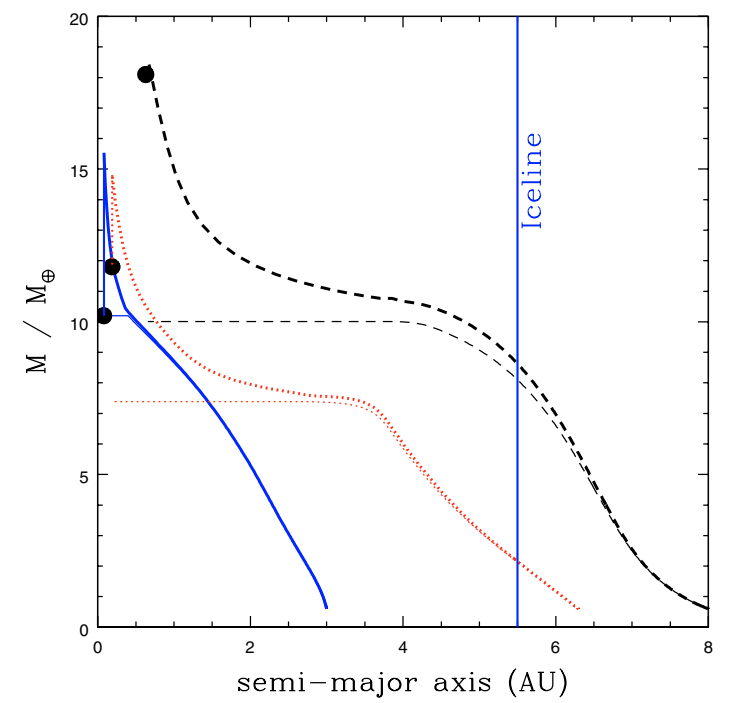

Fig. 1. Formation/evolutionary tracks of the planetary system orbiting HD 69830. The total mass (thick lines) and the core mass (thin lines) for the three planets are given as a function of semi-major axis which is decreasing with time as a result of migration. The iceline is indicated by the vertical line. The minimum mass and semi-major axis derived from the observations are indicated as big dots. The solid lines correspond to the innermost planet, the dotted lines to the middle one, and the dashed lines to the outermost one. The vertical lines at $0.08 \mathrm{AU}$ and $0.18 \mathrm{AU}$ reflect the evaporation of the two innermost planets during 4-10 Gyr, the estimated age of the system.

its Kelvin-Helmholtz timescale (Ida \& Lin 2004). Note that the absence of solids in the innermost regions of the disk (inside $\sim 0.35 \mathrm{AU}$ ), where the temperature exceeds the evaporation temperature of silicates $(\sim 1600 \mathrm{~K})$, leads to a similar effect.

The formation and evolutionary tracks of the three planets in a mass versus semi-major axis diagram are shown in Fig. 1. We found that, in order to reproduce the mass and the semi-major axis of these three planets, gas disks surface densities around $800 \mathrm{~g} / \mathrm{cm}^{2}$ at $5 \mathrm{AU}$ are required. This corresponds to a disk mass (between $0.07 \mathrm{AU}$ and $30 \mathrm{AU}$, and assuming an initial power law for the disk surface density $\Sigma \propto r^{-3 / 2}$ ) of $0.07 M_{\odot}$ and disk lifetime of about $2 \mathrm{Myr}$, both values compatible with values inferred from observations (Haisch et al. 2001; Beckwith \& Sargent 1996). The innermost planet starts well inside the iceline and grows by accreting essentially rocky planetesimals and gas. On the contrary, the two outermost planets start beyond the iceline, and accrete a significant amount of icy planetesimals (as well as gas and rocky planetesimals).

Planet b's embryo starts at $3 \mathrm{AU}$, and, at the time it enters the innermost regions of the disk (below $0.35 \mathrm{AU}$ ), the accretion rate of solids drops dramatically triggering the accretion of gas. The planet reaches its final position at the time the disk vanishes and consists, at the end of the formation process, of a solid core of $\sim 10 M_{\oplus}$ surrounded by an envelope of $\sim 5 M_{\oplus}$. Planet c's embryo starts at $6.5 \mathrm{AU}$ and accretes planetesimals until it enters the region already depleted by the innermost planet ( $3 \mathrm{AU})$. Again, this depletion triggers the accretion of gas, leading to a planet consisting in a rocky/icy core of $\sim 7.5 M_{\oplus}\left(\sim 5.7 M_{\oplus}\right.$ of rocks and $\sim 1.7 M_{\oplus}$ of ices, assuming a standard ices-to-rocks ratio of 4 ) and a $\mathrm{H} / \mathrm{He}$ gaseous envelope of $\sim 7.5 M_{\oplus}$. Finally, planet d's embryo starts at $\sim 8 \mathrm{AU}$ (well beyond the iceline) and accretes a large amount $(\sim 60 \%)$ of icy planetesimals. At the time the growing planet enters the region of the disk already depleted by the second planet, gas accretion is again triggered. The final 
planet consists, at the end of the formation process, of a $\sim 10 M_{\oplus}$ core $\left(\sim 5.2 M_{\oplus}\right.$ of rocks and $\sim 4.8 M_{\oplus}$ of ices), surrounded by a gaseous envelope of $\sim 8 M_{\oplus}$.

Starting from these three formation models, we have then followed the evolution of the three planets taking into account evaporation and irradiation effect (Baraffe et al. 2004, 2006) during 4 to $10 \mathrm{Gyr}$, which corresponds to the estimated age of the HD 69830 system.

\section{Evolution and evaporation}

For this calculation, we took the initial internal compositions obtained at the end of the formation phase. The cores of the two innermost planets are assumed to be made of dunite $\left(\mathrm{Mg}_{2} \mathrm{SiO}_{4}\right.$ - the amount of ice in the core of the middle planet is negligible for the evolution calculation). For the outermost one, we have considered the two limiting cases, one with a pure icy core, and one with a pure rocky core. Each of the three planets is surrounded by a hydrogen/helium envelope whose mass is provided by the formation model. We have taken into account the effect of the incident radiation of the parent star, which modifies the internal structure and the cooling rate of close-in planets, as well as the mass loss due to the evaporation of the outermost layers of the planet's envelope heated by the incident stellar high energy flux. The evaporation rate was chosen to be $1 / 20$ the maximal escape rate of Lammer et al. (2003), a value obtained by various recent detailed hydrodynamical calculations (Tian et al. 2005; Yelle 2004), and consistent with lower limits inferred from observations (Vidal-Madjar et al. 2003).

The effect of irradiation and evaporation is found to be completely negligible for the planet $\mathrm{d}$ and to lead to only a small $(5 \%-10 \%)$ mass loss for planet c (see vertical line at $0.18 \mathrm{AU}$ in Fig. 1). For planet b, however, it is significant (vertical line at 0.08 AU in Fig. 1). Within a few Gyr, essentially all its envelope is evaporated, leaving behind a solid core with only a tiny (less than $2 M_{\oplus}$ ) gaseous atmosphere. Calculations done with slightly different initial conditions (core and envelope mass), yield similar results. The radius of a rocky core (dunite) of $10 M_{\oplus}$ is $0.18 R_{\mathrm{J}}$ (radius of Jupiter). which gives a lower limit for the expected radius of this planet, In the case it was able to retain even a tiny atmosphere its radius will be larger: for an envelope mass of $\sim 2 M_{\oplus}$, the radius would be increased to $\sim 0.45 R_{\mathrm{J}}$. The radii of the two outermost planets are found to be $50-60 \% R_{\mathrm{J}}$, depending on the precise composition of the planet's core and the age of the system.

Figure 2 shows the thermodynamical conditions inside planets $\mathrm{c}$ and $\mathrm{d}$, after $4 \mathrm{Gyr}$, the minimum age of the system, (the results after 10 Gyr are very similar), together with a simplified phase diagram of water. For these two planets, the temperature and pressure are such that water is likely to exist under the form of a super-critical fluid. However, note that no experimental data regarding melting of water are available at the high temperatures characteristic of the planet's interiors.

\section{Conclusions}

We have presented calculations which provide a fully consistent scenario for the formation and evolution of the planetary system around HD 69830. From the calculations presented we can infer the following general scenario for the formation of the system. All three planets start by accreting planetesimals and very little gas as they migrate inwards until they reach a region depleted in solids either by the passage of a previous planet or because of too high temperatures. The main heating source being

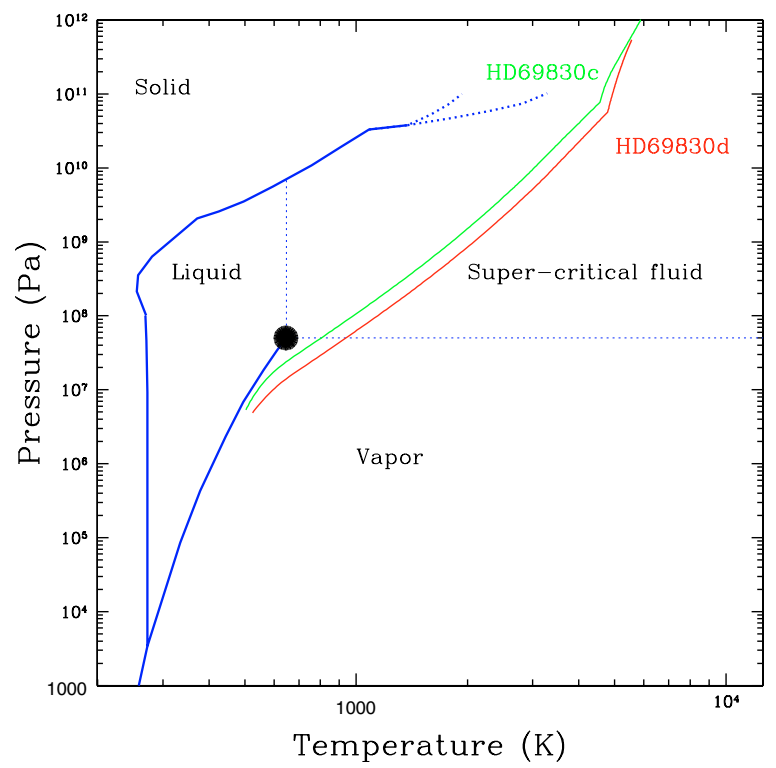

Fig. 2. Thermodynamical conditions inside planets $c$ and $d$ and simplified phase diagram of water. The big dot indicates the position of the critical point. The two heavy dotted curves at high pressures give the likely location of the melting curve (Lin et al. 2005). The thermodynamical conditions are calculated after $4 \mathrm{Gyr}$, assuming a dunite core for HD 69830c, and a ice core for HD 69830d. The kink around $T \sim 4000-5000 \mathrm{~K}$ in the two internal profiles indicates the envelope/core transition (the core is characterised by higher pressures). The two planets harbour a similar structure: a central rocky core, surrounded by a shell of super-critical fluid water, and a hydrogen/helium gas envelope. The results after $10 \mathrm{Gyr}$, or assuming a dunite core for HD 69830d are similar and are not presented for clarity.

suppressed they essentially accrete gas at a rate given by their Kelvin-Helmholtz (KH) timescale (Ida \& Lin 2004). To remain of Neptune-mass without requiring unlikely timing with the disapearence of the disk, a given planet must enter this depleted region when its $\mathrm{KH}$ timescale is of the order of the lifetime of the disk which corresponds to a mass of order 8-12 $M_{\oplus}$ (Ida \& Lin 2004). For the three planets to collect this mass of heavy elements implies a significant amount of migration of the growing cores.

A question which naturally arises with such a planet formation model, is the degree of fine-tuning of the initial conditions needed to produce a planetary system with similar properties (an exact match is meaningless). In this regard, the protoplanetary disk mass and lifetime we require are typical of observed values (Haisch et al. 2001; Beckwith \& Sargent 1996). In fact, the major constraint comes from the fact that the planets, in order to remain of small mass, must enter the planetesimal depleted region of the disk at a time when their accretion timescale (roughly the core's Kelvin-Helmholtz timescale) is comparable to the lifetime of the disk. For the three planets (b, c and d), this timescale is around $1 \mathrm{Myr}, 0.6 \mathrm{Myr}$ and 0.2 Myr. In our simulation the lifetime of the disk is of order 2 Myr. Hence, it is only for the second and third planet (c and d) that this requirement is really limiting the possibilities, but it is certainly not fine-tuning.

For the first time, our consistent formation/evolution calculations lead to the determination of the bulk composition and the inner structure of three Neptune-mass planets: the innermost one consists of a rocky core, with possibly a tiny gaseous envelope, whereas the two outermost planets are made of a central rocky core, a shell of super-critical fluid water and a gaseous envelope. A clear test of the present formation and evolution scenario could 
be achieved by the determination of the mean density of the planets. This would only be possible if the system is seen edgeon and transits are detected so as to measure the radius of the planets. While difficult from the ground, such observations are within reach of HST, COROT or KEPLER. Even if the present system does not lead to observable transits, it is likely that similar, transiting Neptune-mass systems will be discovered in a near future. Confrontation of the present theory with such observations will improve dramatically our understanding of planet formation.

Finally, Spitzer observations of the HD 69830 system have revealed the presence of micron sized dust at distances lower than 1 AU from the central star, that could result from the presence of an asteroid belt (Beichman et al. 2005). Preliminary order of magnitude estimates have shown that the passage of the two inner planets during their formation may significantly but not completely deplete the asteroid belt. Hence, the belt, if present prior to the formation of the planets, would be able to survive at least in part. Interestingly, we note that dust is observed in regions in mean motion resonances with the outermost planet (1:2 and 1:3), that may excite the asteroids, leading to collisions and dust production.
Acknowledgements. This work was supported in part by the Swiss National Science Foundation.

\section{References}

Adams, F. C., Hollenbach, D., Laughlin, G., \& Gorti, U. 2004, ApJ, 611, 360 Alibert, Y., Mordasini, C., Benz, W., \& Winisdoerffer, C. 2005a, A\&A, 434, 343 Alibert, Y., Mousis, O., Mordasini, C., \& Benz, W. 2005b, ApJ, 626, L57 Baraffe, I., Selsis, F., Chabrier, G., et al. 2004, A\&A, 419, L13

Baraffe, I., Alibert, Y., Chabrier, G., \& Benz, W. 2006, A\&A, 450, 1221

Beckwith, S. V. W., \& Sargent, A. I. 1996, Nature, 383, 139

Beichman, C. A., Bryden, G., Gautier, T. N., et al. 2005, ApJ, 626, 1061 Boss, A. P. 2001, ApJ, 563, 367

Boss, A. P. 2006, MNRAS, in press

Haisch, K. E., Lada, E. A., \& Lada, C. J. 2001, ApJ, 553, L153

Hubickyj, O., Bodenheimer, P., \& Lissauer, J. J. 2005, Icarus, 179, 415

Ida, S., \& Lin, D. N. C. 2004, ApJ, 604, 388

Johnstone, D., Hollenbach, D., \& Bally, J. 1998, ApJ, 499, 758

Lammer, H., Selsis, F., Ribas, I., et al. 2003, ApJ, 598, L121

Lin, et al. 2005, GRL, 32, 11306

Lovis, C., Mayor, M., Pepe, F., et al. 2006, Nature, 441, 305

Mayor, M., \& Queloz, D. A. 1995, Nature, 378, 355

Sato, B., Fischer, D. A., Henry, G. W., et al. 2005, ApJ, 633, 465

Tian, F., Toon, O. B., Pavlov, A. A., \& De Sterck, H. 2005, ApJ, 621, 1049

Vidal-Madjar, A., Lecavelier des Etangs, A., Désert, J.-M., et al. 2003, Nature, 422,143

Yelle, R. V. 2004, Icarus, 170, 167 